Physics

Physics Research Publications

\title{
Asymmetric exchange between electron spins in coupled semiconductor quantum dots
}

\author{
S. C. Badescu \\ Y. B. Lyanda-Geller
}

T. L. Reinecke 


\title{
Asymmetric exchange between electron spins in coupled semiconductor quantum dots
}

\author{
Ș. C. Bădescu, ${ }^{1}$ Y. B. Lyanda-Geller, ${ }^{1,2}$ and T. L. Reinecke ${ }^{1}$ \\ ${ }^{1}$ Naval Research Laboratory, Washington, DC 20375, USA \\ ${ }^{2}$ Department of Physics, Purdue University, West Lafayette, Indiana 47907, USA
}

(Received 18 July 2005; published 12 October 2005)

\begin{abstract}
We obtain a microscopic description of the interaction between electron spins in bulk semiconductors and in pairs of semiconductor quantum dots. Treating the $\mathbf{k} \cdot \hat{\mathbf{p}}$ band mixing and the Coulomb interaction on the same footing, we obtain in the third order an asymmetric contribution to the exchange interaction arising from the coupling between the spin of one electron and the relative orbital motion of the other. This contribution does not depend on the inversion asymmetry of the crystal and does not conserve the total spin. We find that this contribution is $\sim 10^{-3}$ of the isotropic exchange, and is of interest in quantum information. Detailed evaluations of the asymmetric exchange are given for several quantum dot systems.
\end{abstract}

DOI: 10.1103/PhysRevB.72.161304

PACS number(s): 73.21.-b, 73.23.Ad, 68.65.Hb, 71.55.Eq

Spins in quantum dots (QDs) are attractive candidates for qubits in quantum information in part because of their long coherence times. ${ }^{1}$ Controllable coupling between these spins is an essential requirement for two qubit quantum gates, and it has been the focus of much recent research. ${ }^{2-4}$ Typically the spin-spin coupling is dominated by the symmetric exchange interaction $J \mathbf{S}_{1} \cdot \mathbf{S}_{2}$, which arises from the Coulomb interaction and the Pauli principle. The symmetry of this part of the exchange with respect to spin permutation implies that the total spin is conserved. This is important in gate operations, which involve pulsing $J(t) .^{5}$

In general, spin-orbit coupling in solids also gives rise to terms that are asymmetric between the spins, and which do not conserve the total spin. These terms can cause loss of fidelity in gate operations involving two spins. To date, the asymmetric terms that have been discussed involve spinorbit coupling of individual electrons in the conduction band. 6,7 For systems with bulk inversion asymmetry, this coupling has the Dresselhaus form. ${ }^{8,9}$ In addition, heterostructure asymmetry can introduce the so-called Rashba coupling. ${ }^{10}$ These two couplings can give an additional interaction between the spins of the Dzyaloshinskii-Moryia (DM) form $\boldsymbol{\beta}_{s o} \cdot\left(\mathbf{S}_{1} \times \mathbf{S}_{2}\right) / \sqrt{\beta_{s o}^{2}+J^{2}}$ (Refs. 11 and 12) where $\boldsymbol{\beta}_{s o}$ is linear in the spin-orbit coupling strength. ${ }^{6,7}$ In general the dephasing caused by contributions of the DM form cannot be totally eliminated, but suggestions have been made for gate pulse shaping that eliminate it to the first order. ${ }^{13}$

Here we give a different contribution to the spin-spin coupling of two electrons in III-V semiconductors that does not require either bulk inversion asymmetry or Rashba coupling. It arises from the Coulomb interaction between two electrons and from the conduction-valence band mixing. It is similar to the exchange interaction between excitons. ${ }^{14}$ We describe this asymmetric electron spin coupling in bulk materials, and we make detailed evaluations for spins in several coupled QD systems.

We use a $8 \times 8$ Kane Hamiltonian to represent the band structure of III-V semiconductors, ${ }^{14}$ with a gap between an $s$-like conduction band and $p$-like valence bands. The band coupling in this Hamiltonian is obtained in the $\mathbf{k} \cdot \hat{\mathbf{p}}$ effective mass perturbation theory, where $\hat{\mathbf{p}}$ is the relevant momentum operator. The standard parameters in this approach are the band gap $E_{g}$, the energy of the split-off band $\Delta$, and the valence-conduction band coupling $P=\left(\hbar / m_{0}\right)\left\langle S\left|\hat{p}_{x}\right| X\right\rangle$, where $|S\rangle$ and $|X\rangle$ are the Bloch states of the conduction and valence bands. ${ }^{15}$

We consider two electrons of relative coordinate $\mathbf{r}=\mathbf{r}_{1}$ $-\mathbf{r}_{2}$ in a semiconductor of dielectric permittivity $\kappa$. The unperturbed two-particle Hamiltonian in the conduction band is $H^{(0)}=\left(\hat{\mathbf{p}}_{1}^{2}+\hat{\mathbf{p}}_{2}^{2}\right) / 2 m_{0}$. The two band mixing terms $\mathbf{k}_{1} \cdot \hat{\mathbf{p}}_{1}$, $\mathbf{k}_{2} \cdot \hat{\mathbf{p}}_{2}$, and the Coulomb interaction $U_{C}=e^{2} / \kappa \mathbf{r}$ between the electrons are treated on equal footing. The leading part of the spin-dependent interaction arises in third order from first order contributions of $U_{C}$ and of each of the band mixing terms $\mathbf{k}_{1} \cdot \hat{\mathbf{p}}_{1}, \mathbf{k}_{2} \cdot \hat{\mathbf{p}}_{2}$. The spin-dependent part of the Coulomb interaction arises from the coupling between the electron spins and their relative motion and is:

$$
\begin{aligned}
H_{s}^{(3)}= & -\frac{e^{2}}{\kappa} \frac{2 P^{2}}{3 E_{g}^{2}} \frac{\Delta\left(2 E_{g}+\Delta\right)}{\left(E_{g}+\Delta\right)^{2}} \\
& \times\left[\left(\mathbf{r} \times \hat{\mathbf{p}}_{1}\right) \cdot \mathbf{S}_{1}-\left(\mathbf{r} \times \hat{\mathbf{p}}_{2}\right) \cdot \mathbf{S}_{2}\right] / \hbar^{2} r^{3} .
\end{aligned}
$$

The coefficient of the square bracket in Eq. (1) is the coupling constant, which we will call $C$. For GaAs $C$ $=5.7 \mathrm{meV} \mathrm{nm}^{3}$, and for InAs $C=10 \mathrm{meV} \mathrm{nm}^{3}$. The remaining spin-independent terms in this order of the theory contribute to the isotropic exchange $J$. These include a "local contact" term of the form

$$
H_{c}^{(3)}=-\frac{4 \pi e^{2} P^{2}}{3 \kappa} \frac{\left(E_{g}+\Delta\right)^{2}+E_{g}^{2}}{E_{g}^{2}\left(E_{g}+\Delta\right)^{2}} \delta(\mathbf{r}) .
$$

Coupling terms similar to $H_{s}^{(3)}$ and $H_{c}^{(3)}$ are known for two electrons in the free space ${ }^{16}$ where they arise from electronpositron band mixing and Coulomb interaction. In the present case the effect is stronger because the energy gap $E_{g}$ between electrons and holes in a crystal is much smaller than the energy gap $m_{0} c^{2}$ between electrons and positrons. Also, the symmetry of interaction $H_{s}^{(3)}$ [Eq. (1)] is different from that between electrons in free space ${ }^{16}$ because the valence bands $\Gamma_{8}$ and $\Gamma_{7}$ have a symmetry different from the $s$ symmetry of positrons. 
We note that for itinerant electrons the interaction $H_{s}^{(3)}$ [Eq. (1)] flips a spin in an electron-electron collision, providing a mechanism for the relaxation of spin polarization in addition to other known mechanisms. ${ }^{17}$ We evaluated the spin dephasing time for a two-dimensional (2D) electron gas in GaAs quantum wells and found that it is of the order $\sim 1 \mathrm{~ns}$ for He temperature and of the order $\sim 1 \mathrm{ps}$ for room temperatures. This suggests that the interaction $H_{s}^{(3)}$ can be important in spin transport in low-dimensional structures, such as quantum wells. ${ }^{18}$

Here we will primarily address systems with spins on two centers, such as two QDs or two charged donors, where $H_{s}^{(3)}$ gives rise to a DM coupling. The two electrons can be in a singlet state with total spin $S=0$ or in a triplet state with total spin $S=1 . H_{s}^{(3)}$ has nonzero matrix elements between states of different total spin. We take the lowest singlet and triplet states to be separated by an exchange energy $J$. Then in the Hilbert space of these two states the Hamiltonian can be written in the form

$$
\tilde{H}=J \mathbf{S}_{1} \cdot \mathbf{S}_{2}+i \boldsymbol{\beta} \cdot\left(\mathbf{S}_{1}-\mathbf{S}_{2}\right),
$$

where $i \boldsymbol{\beta}=-C / \hbar^{2}\left\langle S_{0}\left|\left(\mathbf{r} / r^{3}\right) \times \mathbf{p}_{1}\right| T_{0}\right\rangle$ is the matrix element between the lowest orbital singlet $\left|S_{0}\right\rangle$ and the triplet $\left|T_{0}\right\rangle$. A block diagonal form equivalent to Eq. (3) can be obtained by an orthogonal transformation in spin space, giving the so called "twisted spin representation"19

$$
\begin{aligned}
\tilde{H}= & J \cos \phi \mathbf{S}_{1} \cdot \mathbf{S}_{2}+2 J\left(\sin \frac{\phi}{2}\right)^{2}\left(\mathbf{n} \cdot \mathbf{S}_{1}\right)\left(\mathbf{n} \cdot \mathbf{S}_{2}\right) \\
& +J \sin \phi \mathbf{n} \cdot\left(\mathbf{S}_{1} \times \mathbf{S}_{2}\right),
\end{aligned}
$$

where the spin-0 and spin-1 states are mixed by the operator $\exp \left[i(\phi / 2) \mathbf{n} \cdot\left(\mathbf{S}_{1}-\mathbf{S}_{2}\right)\right]$, with $\mathbf{n}=\boldsymbol{\beta} / \beta$ and $\phi=\arctan (\beta / J)$. In Eq. (4) the first two terms are the symmetric isotropic and the symmetric anisotropic Heisenberg terms. The last term is the asymmetric exchange now in the DM form $\boldsymbol{\beta} \cdot\left(\mathbf{S}_{1}\right.$ $\left.\times \mathbf{S}_{2}\right) / \sqrt{\beta^{2}+J^{2}}$. The contributions from the Dresselhaus and Rashba couplings to the anisotropic and asymmetric exchange were given in the form of Eq. (4) in Ref. 6.

We consider the lowest single-particle states $\left|\varphi_{ \pm}\right\rangle$from the two confining centers. In the absence of spin-orbit coupling the two-particle wave functions can be written as products of orbital states and spin states. The axial vector $\boldsymbol{\beta}$ is nonzero only for inversion-asymmetric confining potentials. For such systems we take into account the possibility that both electrons occupy the same site, and therefore we use the HundMulliken description of the two-particle orbital states. ${ }^{4} \mathrm{We}$ obtain the ground state by diagonalizing analytically the tunnelling and the Coulomb and local contact interactions. We then focus on the Hilbert space determined by the lowest singlet $(S=0)$ and triplet states $(S=1)$ in which the Hamiltonian is given by Eq. (3). The importance of the resulting $\mathrm{DM}$ asymmetric exchange depends on the ratio $\tan \phi=\beta / J$ between the coefficients of the third and the first terms in Eq. (4).

Consider first the ratio $\beta / J$ for electrons confined by two vertically coupled QDs, such as to those in InAs/GaAs systems. ${ }^{20}$ These dots generally have different sizes and can have shape asymmetries as well. ${ }^{21}$ The conduction band offset between GaAs and InAs $\left(U_{0} \sim 0.7 \mathrm{eV}\right)$ is larger than the quantization energy in the lateral direction $(\hbar \omega \sim 20 \mathrm{meV})$ by more than an order of magnitude. This allows us to decouple the vertical and lateral degrees of freedom. We describe these dots by the potential offsets along the growth axis $z$ and parabolic potentials in lateral directions $x$ and $y$, which in general can be anisotropic. These lateral directions are independent of the crystal axes. The potentials and wave functions then can be written straightforwardly. ${ }^{22,23}$ Thus, the asymmetry studied here results from differences in the lateral sizes and shapes of the QDs.

The dependence of the ratio $\beta / J$ of the asymmetric exchange to the symmetric exchange on the separation $d$ between the two cylindrical dots with a fixed lateral offset $b$ is shown in Fig. 1(a). Its dependence on the lateral offset $b$ for two dots at a fixed $d$ is shown in Fig. 1(b). The insets of Figs. 1 (a) and 1(b) show sketches of the lateral and vertical views of two cylindrical dots with vectors $\boldsymbol{b}, \boldsymbol{d}$ pointing from the smaller dot to the larger one. The vector $\boldsymbol{\beta}$ is oriented in the direction $\boldsymbol{b} \times \boldsymbol{d}$, and for small differences $\Delta a=a_{+}-a_{-}$between the dot radii $a_{ \pm}$, it is proportional to $\Delta a$. It follows from Eq. (1) that for two cylindrical dots of equal sizes, $\beta / J$ vanishes because the system has a center of inversion $(\boldsymbol{d}$ is not defined). For any two cylindrical dots without a lateral offset, $\beta / J$ also vanishes because the ground state has cylindrical symmetry $(\boldsymbol{b} \times \boldsymbol{d}=0)$. In each of these figures there are two regions of behavior: (i) For small size differences $\Delta a$ (the left sides of the peaks) $\beta / J$ increases roughly proportional to $d$ in Fig. 1(a) and roughly proportional to $b$ in Fig. (b) until it reaches $\sim 3.5 \times 10^{-3}$; in this region the two electrons are distributed almost symmetrically between the dots. (ii) For larger values of $\Delta a$ (right sides of the peaks), $\beta / J$ first increases with $d$ to a small maximum and then decreases [Fig. 1(a)], and similarly with $b$ Fig. 1(b)]. In this second region both electrons tend to occupy preferentially the larger dot, where the Coulomb energy is overcome by the difference between single-particle energies of the two dots.

In order to study shape asymmetry, we consider two vertically coupled dots with deviations from cylindrical symmetry. In Fig. 2 we give results for two identical elliptical dots that are rotated by $\pi / 2$ with respect to one another, as shown in the inset, with an offset $b=4 \mathrm{~nm}$. The dots of equal sizes have equal energies, which leads to an equal distribution of two electrons on them. The ratio $\beta / J$ reaches substantial values $\left(\sim 10^{-3}\right)$ as a function of the separation $d$ and has a relatively weak dependence on the angle $\gamma$ between the axes of the dots. In this case $\beta$ arises from the shape asymmetry. The coupling depends on the angle $\gamma$ between the relative position vector and the principal axes (for cylindrical dots, $\boldsymbol{\beta}$ changes direction but is constant in magnitude). The orientation of $\boldsymbol{\beta}$ is given by $\boldsymbol{b} \times \boldsymbol{d}$. In the case where the axes of the dots are parallel $\left(a_{-, x}=a_{+, x}, a_{-, y}=a_{+, y}\right)$, the asymmetric exchange is zero [the system then has an inversion center at $(\boldsymbol{b} / 2, \boldsymbol{d} / 2)]$. In cases when the dots are different in size and not cylindrical, $\beta / J$ is even larger. For example, for a cylindrical dot with $a_{-}=5 \mathrm{~nm}$ coupled with an elliptical dot with $a_{+, x}=4 \mathrm{~nm}, a_{+, y}=6.25 \mathrm{~nm}$, the maxima of $\beta / J$ are $\sim 3.5$ $\times 10^{-3}$ for all $\gamma$. 

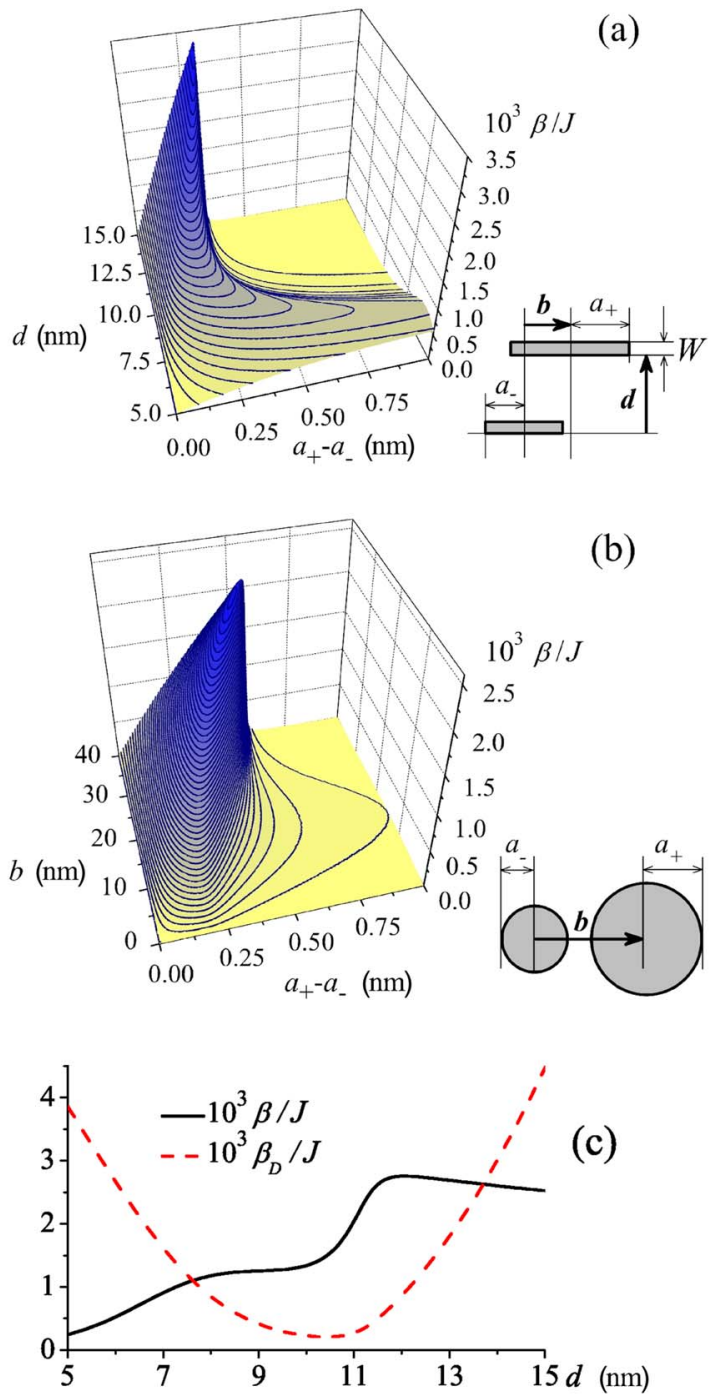

FIG. 1. (Color online) (a) Dependence of the asymmetric exchange $\beta / J$ on the separation $d$ along the growth axes between two cylindrical vertically coupled dots with a lateral offset $b=4 \mathrm{~nm}$, for a smaller dot size $a_{-}=5 \mathrm{~nm}$ and differences between radii of dots $\Delta a=a_{+}-a_{-} \in[5,6] \mathrm{nm}$. (b) dependence of asymmetric exchange on the lateral offset $b$ at a separation $d=10 \mathrm{~nm}$ for $a_{-}=5 \mathrm{~nm}$ and varying $\Delta a$. The insets in $[(\mathrm{a}) /(\mathrm{b})]$ are lateral/vertical views of the geometry. (c) Asymmetric exchange $\beta / J$ obtained here (solid line) compared with the asymmetric exchange $\beta_{D} / J$ from Dresselhaus coupling (dashed line) as a function of separation $d$, for $\Delta a$ $=0.22 \mathrm{~nm}, a_{-}=5 \mathrm{~nm}$, and $b=4 \mathrm{~nm}$.

We have calculated the contribution to the asymmetric exchange for these structures from the bulk Dresselhaus coupling $H_{s o}^{D}=i \gamma_{s o}^{D} \partial_{x}\left(\partial_{y}^{2}-\partial_{z}^{2}\right) S_{\alpha} / \hbar$ (plus cyclic permutations of cartesian indices). The coupling constant $\gamma_{s o}^{P}$ is $47 \mathrm{meV} \mathrm{nm}^{3}$ for GaAs, and for InAs it is of the order of $100 \mathrm{meV} \mathrm{nm}^{3}$. In Fig. 1(c) the contribution $\beta_{D} / J$ from the Dresselhaus coupling to the asymmetric exchange is compared with the asymmetric exchange $\beta / J$ for a size difference $\Delta a$ $=0.22 \mathrm{~nm} \cdot{ }^{24-26}$ We see that the asymmetric contribution $\beta / J$ presented here is larger for intermediate separations $d$, a region of particular interest for implementations for quantum

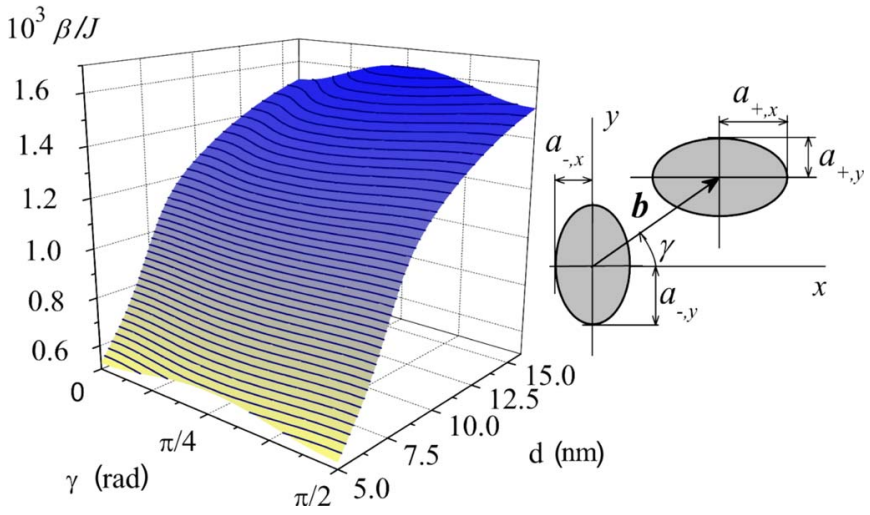

FIG. 2. (Color online) Dependence of modulus of asymmetric exchange $\beta / J$ on angle $\gamma$ between the axes of two elliptical vertically coupled dots of equal major and minor axes $a_{-, x}=a_{+, y}$ $=3.5 \mathrm{~nm}, a_{-, y}=a_{+, x}=6.5 \mathrm{~nm}$, at $b=4 \mathrm{~nm}$. The inset gives a projection of the dot contours on a plane perpendicular to the growth axis, using the harmonic potential parameters to represent the dot sizes.

information. As the difference in the dot sizes increases, $\beta / J$ becomes larger relative to $\beta_{D} / J$, and as the size difference decreases, $\beta_{D} / J$ becomes larger.

We have also considered laterally coupled QDs. To obtain a representation of the barrier between the QDs we use inverted-Gaussian potentials, ${ }^{27}$ and we use again the material parameters for InAs. The wave functions are obtained variationally. ${ }^{28}$ In Fig. 3 we give results for two elliptical dots of equal sizes rotated with respect to each other by $\pi / 2$. In this case the anisotropic exchange $\beta / J$ arises from the shape asymmetry. From the operator $\mathbf{r} \times \nabla_{1}$ in Eq. (1), the asymmetric exchange $\beta$ has a nonzero component only along the growth axis, and the dependence of the modulus $\beta / J$ is symmetric with respect to $\gamma=\pi / 4$. In Fig. 3 once again $\beta / J$ reaches a maximum of $\sim 10^{-3}$. In cases when the Dresselhaus ${ }^{25}$ and Rashba ${ }^{26}$ couplings have equal coupling constants, their contribution is small for $\gamma=\pi / 4$ and then the total asymmetric exchange is dominated by $\beta / J$.

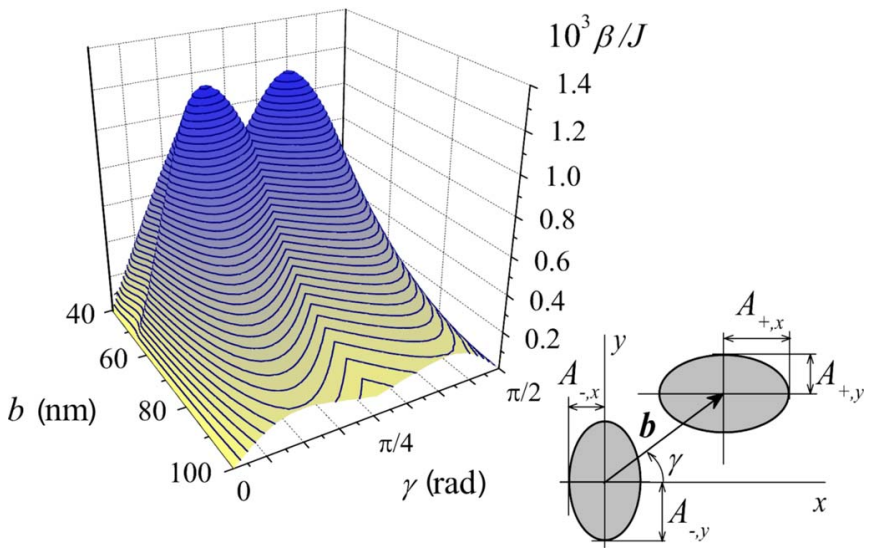

FIG. 3. (Color online) Dependence of the modulus of the asymmetric exchange $\beta / J$ on angle $\gamma$ between the axes of two elliptical laterally coupled dots of equal potential sizes $A_{-, x}=A_{+, y}=6 \mathrm{~nm}$ and $A_{-, y}=A_{+, x}=9 \mathrm{~nm}$. The inset shows the two QDs that lie in the same plane, seen along the growth axis, using the Gaussian potential parameters to define their sizes. 
In summary, we derived an asymmetric contribution to the exchange interaction between two electrons in III-V semiconductors that arises from the Coulomb interaction and the band mixing and which does not require inversion asymmetry. For asymmetric coupled semiconductor QDs, this contribution depends on the geometry and is typically $10^{-3}$ of the isotropic exchange $J$. The asymmetric exchange results in mixing states of different total spin and in loss of fidelity in quantum gates. The magnitude of the exchange obtained here would require corrections in gate pulsing in order to avoid dephasing. ${ }^{4,5}$ This interaction also can play a role in the relaxation and dephasing of spin in transport processes in lowdimensional structures.

This work was supported by the DARPA QUIST program, by ONR and by the ONR Nanoscale Electronics Program. S.C.B. holds a NRC/NRL Associateship Award.
${ }^{1}$ J. A. Gupta, D. D. Awschalom, X. Peng, and A. P. Alivisatos, Phys. Rev. B 59, R10421 (1999).

${ }^{2}$ D. Loss and D. P. DiVincenzo, Phys. Rev. A 57, 120 (1998).

${ }^{3}$ X. Hu and S. Das Sarma, Phys. Rev. A 61, 062301 (2001).

${ }^{4}$ L. A. Wu and D. A. Lidar, Phys. Rev. A 66, 062314 (2002).

${ }^{5}$ D. Stepanenko, N. E. Bonesteel, D. P. DiVincenzo, G. Burkard, and D. Loss, Phys. Rev. B 68, 115306 (2003).

${ }^{6}$ K. V. Kavokin, Phys. Rev. B 64, 075305 (2001).

${ }^{7}$ L. P. Gor'kov and P. L. Krotkov, Phys. Rev. B 68, 155206 (2003).

${ }^{8}$ G. Dresselhaus, Phys. Rev. 100, 580 (1955).

${ }^{9}$ B. L. Altshuler, A. G. Aronov, A. I. Larkin, and D. E. Khmelnitskii, Sov. Phys. JETP 54, 411 (1981).

${ }^{10}$ Yu. L. Bychkov and E. I. Rashba, JETP Lett. 39, 78 (1984).

${ }^{11}$ I. E. Dzyaloshinskii, Phys. Chem. Solids 4, 241 (1958).

${ }^{12}$ T. Morya, J. Phys. Chem. Solids 120, 91 (1960).

${ }^{13}$ G. Burkard and D. Loss, Phys. Rev. Lett. 88, 047903 (2002).

${ }^{14}$ G. L. Bir and G. E. Pikus, Symmetry and Strain-Induced Effects in Semiconductors (Wiley, New York, 1974).

${ }^{15}$ The Kane parameters used here are: $\Delta=0.39 \mathrm{eV}, E_{g}$ $=0.42 \mathrm{eV}, 2 P^{2} / m_{0}=21.5 \mathrm{eV}$ for InAs and $\Delta=0.417 \mathrm{eV}, E_{g}$ $=0.39 \mathrm{eV}, 2 P^{2} / m_{0}=28.8 \mathrm{eV}$ for GaAs, where $m_{0}$ is the free electron mass. The conduction band of III-V semiconductors has symmetry $\Gamma_{6}$ and the valence bands have symmetries $\Gamma_{7}$ and $\Gamma_{8}$.

${ }^{16}$ V. B. Berestetskii, E. M. Lifshitz, and L. P. Pitaevskii, Quantum Electrodynamics (Butterwort-Heinmann, Oxford, 2002), Chap. IX (Breit-Landau Equation).

${ }^{17}$ F. Meier and B. P. Zakharchenya, Optical Orientation (Elsevier Science, New York, 1984).

${ }^{18}$ Y. K. Kato, R. C. Myers, A. C. Gossard, and D. D. Awschalom, Phys. Rev. Lett. 93, 176601 (2004).

${ }^{19}$ L. S. Levitov and E. I. Rashba, Phys. Rev. B 67, 115324 (2003).

${ }^{20}$ W. Seifert, N. Carlsson, M. Miller, M. E. Pistol, L. Samuelson, and L. R. Wallenberg, Prog. Cryst. Growth Charact. Mater. 33, 423 (1996).
${ }^{21}$ R. Krebs, S. Deubert, J. P. Reithmaier, and A. Forchel, J. Cryst. Growth 251, 742 (2003).

${ }^{22}$ The QDs are centered at $\pm\left(b_{x} / 2, b_{y} / 2, d / 2\right)$. Using $x_{ \pm}$ $=x \mp b_{x} / 2, y_{ \pm}=y \mp b_{y} / 2, z_{ \pm}=z \mp d / 2$ for the two dots $(+/-)$, the single-dot potentials are (Ref. 23) $U_{ \pm}^{v}=\left[m\left(\omega_{ \pm, x}^{2} x_{ \pm}^{2}+\omega_{ \pm, y}^{2} y_{ \pm}^{2}\right) / 2\right.$ $\left.-U_{0}\right] W \delta\left(z_{ \pm}\right)$. For each separate dot, the lowest one-particle electronic state is $\varphi_{ \pm}^{0}=\sqrt{\left(q / 4 \pi a_{ \pm, x} a_{ \pm, y}\right)} \exp \left[-\left(x_{ \pm} / 2 a_{ \pm, x}\right)^{2}-\left(y_{ \pm} /\right.\right.$ $\left.\left.2 a_{ \pm, y}\right)^{2}-q\left|z_{ \pm}\right| / 2\right]$. The lateral sizes of $\varphi_{ \pm}^{0}$ are $a_{ \pm, \alpha}=\sqrt{\hbar / 2 m \omega_{ \pm, \alpha}}$ and the vertical size is $q=2 m_{b} W U_{0} / \hbar^{2}$, where: $\alpha=x, y, \hbar \omega_{ \pm, \alpha}$ are the lateral quantization energies and $m=0.023 m_{0}, m_{b}$ $=0.067 m_{0}$ are the electron effective masses in InAs and in the GaAs barrier. The total confining potential is $U_{t}^{v}=U_{+}^{v}+U_{-}^{v}$. The two Wannier states $\left|\varphi_{ \pm}\right\rangle$are obtained by a symmetric orthogonalization of the single-dot orbitals: $\left|\varphi_{ \pm}\right\rangle=\left(\cos (\theta / 2)\left|\varphi_{ \pm}^{0}\right\rangle\right.$ $\left.-\sin (\theta / 2)\left|\varphi_{\mp}^{0}\right\rangle\right) / \cos \theta$. We consider dots of equal thicknesses $W$, and evaluate the lateral potentials from splittings between the lowest states in experiment.

${ }^{23}$ Y. B. Lyanda-Geller, T. L Reinecke, and M. Bayer, Phys. Rev. B 69, 161308(R) (2004).

${ }^{24}$ In general, a structure asymmetry can give rise to a Rashba coupling that can be opposite in sign to the Dresselhaus coupling (see Ref. 25) and thus decrease $\beta_{D} / J$. This contribution is expected to be small for the present system.

${ }^{25}$ W. Knap, C. Skierbiszewski, A. Zduniak, E. Litwin-Staszewska, D. Bertho, F. Kobbi, J. L. Robert, G. E. Pikus, F. G. Pikus, S. V. Iordanskii, V. Mosser, K. Zekentes, and Y. B. Lyanda-Geller, Phys. Rev. B 53, 3912 (1996).

${ }^{26}$ M. J. Yang, C. H. Yang, and Y. B. Lyanda-Geller, Europhys. Lett. 66, 826 (2004).

${ }^{27}$ S. M. Reimann and M. Manninen, Rev. Mod. Phys. 74, 1283 (2002).

${ }^{28}$ The inverted Gaussian potentials $U_{ \pm}^{l}$ of widths $A_{ \pm, \alpha}$ for the two QDs $(+/-)$ and the wave functions are, respectively: $U_{ \pm}^{l}$ $=V_{ \pm} \exp \left[-\left(x_{ \pm} / A_{ \pm, x}\right)^{2}-\left(y_{ \pm} / A_{ \pm, y}\right)^{2}\right], \varphi_{ \pm}^{0}=\exp \left[-\left(x_{ \pm} / 2 a_{ \pm, x}\right)^{2}-\left(y_{ \pm} /\right.\right.$ $\left.\left.2 a_{ \pm, y}\right)^{2}\right] / \sqrt{2 \pi a_{ \pm, x} a_{ \pm, y}}$, where $A_{ \pm, \alpha}$ defines the size of the dots and the wave function parameters $a_{ \pm, \alpha}$ are obtained variationally. 